\title{
Study of Nonradiative Recombination Centers in n-GaN Grown on LT-GaN and AlN Buffer Layer by Below-Gap Excitation
}

\author{
M. D. Haque ${ }^{1,2}$, M. Julkarnain ${ }^{3}$, A. Z. M. Touhidul Islam4, N. Kamata1 \\ ${ }^{1}$ Department of Functional Materials Science, Saitama University, Saitama, Japan \\ ${ }^{2}$ Department of Electronics and Communication Engineering, Hajee Mohammad Danesh Science and Technology University, \\ Dinajpur, Bangladesh \\ ${ }^{3}$ Department of Applied Physics and Electronic Engineering, University of Rajshahi, Rajshahi, Bangladesh \\ ${ }^{4}$ Department of Electrical and Electronic Engineering, University of Rajshahi, Rajshahi, Bangladesh \\ Email: haque.m.d.953@ms.saitam-u.ac.jp,dulalhaque@hstu.ac.bd
}

How to cite this paper: Haque, M.D., Julkarnain, M., Islam, A.Z.M.T. and Kamata, N. (2018) Study of Nonradiative Recombination Centers in $\mathrm{n}-\mathrm{GaN}$ Grown on LT-GaN and AlN Buffer Layer by Below-Gap Excitation. Advances in Materials Physics and Chemistry, 8, 143-155.

https://doi.org/10.4236/ampc.2018.83010

Received: February 26, 2018

Accepted: March 27, 2018

Published: March 30, 2018

Copyright $(9) 2018$ by authors and Scientific Research Publishing Inc. This work is licensed under the Creative Commons Attribution International License (CC BY 4.0).

http://creativecommons.org/licenses/by/4.0/

\begin{abstract}
Nonradiative recombination (NRR) centers in n-type GaN samples grown by MOCVD technique on a LT-GaN buffer layer and aAlN buffer layer have been studied by two wavelength excited photoluminescence (TWEPL). The near band-edge photoluminescence (PL) intensity decreases due to the superposition of below-gap excitation (BGE) light of energies $0.93,1.17$ and 1.27 $\mathrm{eV}$ over above-gap excitation (AGE) light of energy $4.66 \mathrm{eV}$. The decrease in PL intensity due to the addition of the BGE has been explained by a two levels recombination model based on SRH statistics. It indicates the presence of a pair of NRR centers in both samples, which are activated by the BGE. The degree of quenching in PL intensity for the sample grown on LT-GaN buffer layer is stronger than the sample grown on AlN buffer layer for all BGE sources. This result implies that the use of the AlN buffer layer is more effective for reducing the NRR centers in $n-G a N$ layers than the LT-GaN buffer layer. The dependence of PL quenching on the AGE density, the BGE density and temperature has been also investigated. The NRR parameters have been quantitatively determined by solving rate equations and fitting the simulated results with the experimental data.
\end{abstract}

\section{Keywords}

N-Type GaN, Two-Wavelength Excited Photoluminescence, Nonradiative Recombination Center, Recombination Model 


\section{Introduction}

Gallium nitride $(\mathrm{GaN})$ has been developed as a basis semiconductor for InGaN and $\mathrm{AlGaN}$ ternary compounds for such applications as green, blue, up to deep ultra-violet light emitters and high power electronic devices [1]. However, the full potential of $\mathrm{GaN}$ based devices has been restricted due to the lack of suitable substrate [2]. The sapphire substrates are generally used owing to low cost and high temperature stability [3]. But they introduce threading dislocations in a typical range of $10^{9}-10^{11} \mathrm{~cm}^{-2}$ due to lattice and thermal mismatch between epitaxial layer and substrate [4] [5] [6]. High density of these structural defects forms below-gap states in group III-V semiconductors (such as GaAs, InP and $\mathrm{GaN}$ ) which act as non-radiative recombination (NRR) centers in the crystal and degrade the device efficiency and lifetime [7] [8] [9]. The insertion of buffer layer between substrates and epilayers has generated a lot of research interest for decreasing defect density in GaN based optoelectronics and microelectronics devices [10] [11]. It has been reported that the insertion of thin AlN buffer layer between GaN epilayer and sapphire substrate can reduce tensile growth stress and dislocation density which in turn improve crystalline quality compared to that of the LT-GaN buffer layer [12] [13] [14] [15]. Recently, the high temperature AlGaN MSFET with AlN buffer layer and better surface morphology and crystalline quality of thick AlGaN have been realized for the growth on the AlN buffer layer [16] [17]. However, for further improvement of GaN based device performance, it is still insufficient to understand the formation mechanism of defect states and structural optimization for eliminating them during the growth process. The GaN epilayers grown on LT-GaN buffer and AlN buffer layers has been characterized by photoluminescence (PL), scanning electron microscopy (SEM) and atomic force microscopy (AFM) studies [11] [18] [19] [20], but these methods give little information about NRR centers. Deep Level Transient Spectroscopy (DLTS) has been also used to study the deep levels in GaN epilayers [21] but its applications are restricted due to the necessity of preparing suitable sample for the measurements. On the other hand, our two-wavelength excited photoluminescence (TWEPL) is a versatile non-contacting and non-destructive scheme; no need to arrange any special kind of sample preparation. A comparative study of these types of samples has not been reported yet by this method.

In this work, TWEPL has been used for the detection and characterization of NRR centers in n-type GaN layers on a LT-GaN buffer layer and aAlN buffer layer grown on sapphire substrates. The change in PL peak intensity due to the addition of the BGE light over that of the AGE is observed as a function of AGE density, BGE density, and temperature. The NRR parameters have also been evaluated by systematically solving the rate equations and fitting the results with experimental data.

\section{Experimental}

\subsection{Sample Structure}

Two n-type GaN layers with Si concentration of $3 \times 10^{16} \mathrm{~cm}^{-3}$ were grown on 
LT-GaN (sample A) and AlN buffer layer (Sample B), respectively, by metal organic chemical vapor deposition (MOCVD) method. The detailed structure of the samples is shown in Figure 1. The $\mathrm{n}-\mathrm{GaN}(1.7 \mu \mathrm{m})$ layer was grown at $1050^{\circ} \mathrm{C}$ after the sequence of LT-GaN $(30 \mathrm{~nm})$ or AlN $(1.0 \mu \mathrm{m})$ buffer and i-GaN $(3.0 \mu \mathrm{m})$ layer on c-plane (0001) sapphire substrate. All the layers except the buffer layer are same for both samples.

\subsection{Measurement}

The experimental setup for characterizing the $\mathrm{n}-\mathrm{GaN}$ layers by TWEPL is shown in Figure 2. The sample was mounted in a temperature-controlled cryostat and

\begin{tabular}{|c|}
\hline $\mathrm{n}-\mathrm{GaN}(1.7 \mu \mathrm{m})$ \\
\hline i-GaN $(3 \mu \mathrm{m})$ \\
\hline LT-GaN buffer $(30 \mathrm{~nm})$ \\
\hline Sapphire Substrate \\
\hline
\end{tabular}

(a) Sample A

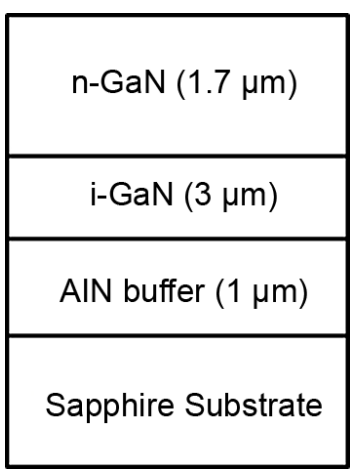

(b) Sample B

Figure 1. Structure of n-type GaN samples; (a) sample A with LT-GaN buffer layer and (b) sample B with AlN buffer layer.

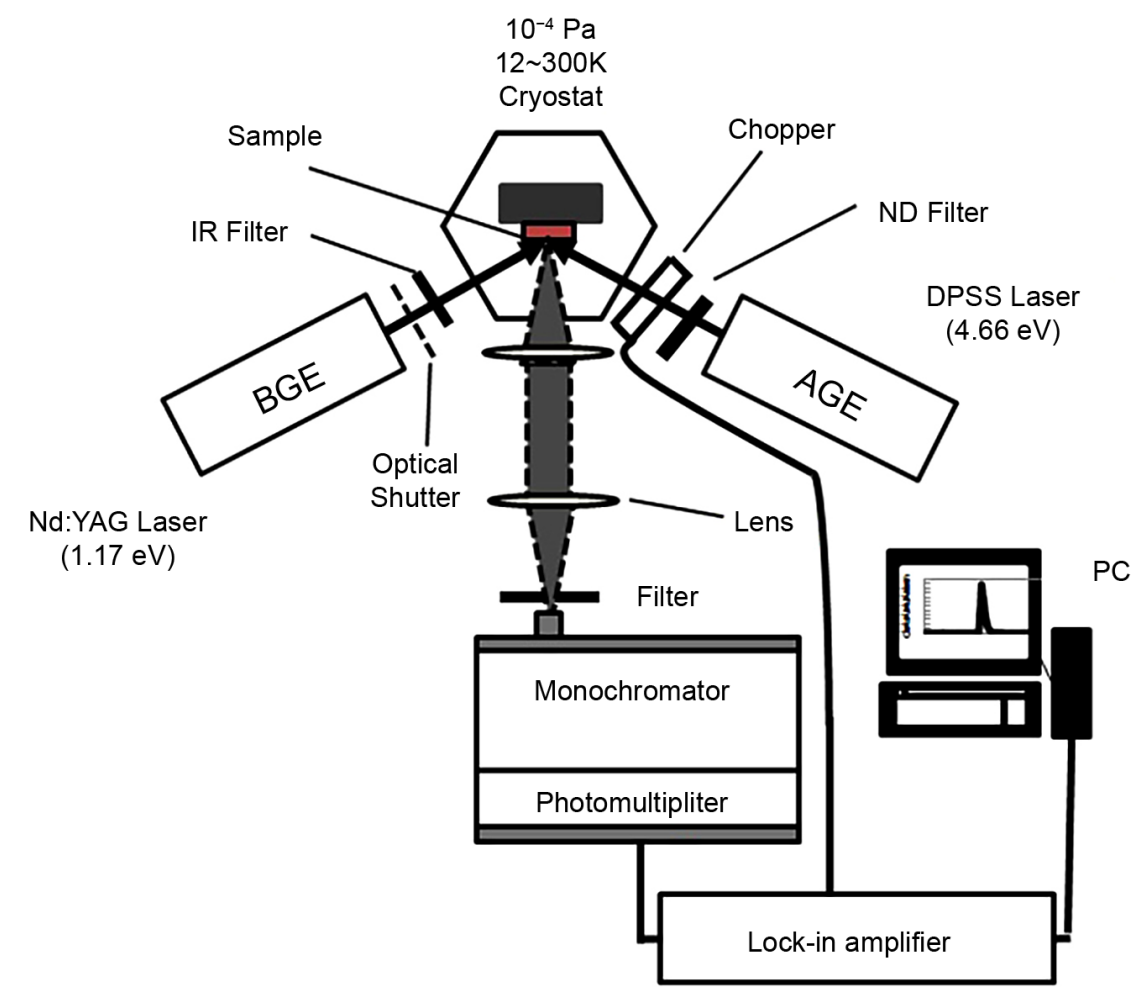

Figure 2. Experimental setup of the TWEPL measurement. 
irradiated by diode-pumped solid-state (DPSS) laser of $4.66 \mathrm{eV}(266 \mathrm{~nm})$ as the AGE. The conventional PL spectra of both samples were measured at a temperature of $12 \mathrm{~K}$. The output power of the AGE laser was kept constant at $16 \mathrm{~mW}$ and the neutral density filters were used for changing the excitation density. A temporally switched BGE light by an optical shutter with the internal 500 seconds ON/OFF was superposed on the AGE light to excite the sample at the same point. The BGE light of energy $1.27 \mathrm{eV}(980 \mathrm{~nm}), 1.17 \mathrm{eV}(1064 \mathrm{~nm})$, and $0.93 \mathrm{eV}(1340 \mathrm{~nm})$ were used for the TWEPL measurement. The modulated PL signal from the sample was fed to a monochromator through a set of objective lenses, converted to photocurrent by a photomultiplier and recorded by a computer after the lock-in amplification. Here, an optical chopper was used with the digital lock-in-amplifier for improving the $\mathrm{S} / \mathrm{N}$ ratio of the PL signal. By measuring PL intensity with and without the BGE, $I_{A G E+B G E}$ and $I_{A G E}$ respectively, the normalized PL intensity $I_{N}=I_{A G E+B G E} / I_{A G E}$ was determined.

\section{Results and Discussion}

\subsection{PL Intensity Comparison}

The PL spectra of $\mathrm{n}-\mathrm{GaN}$ samples measured at $12 \mathrm{~K}$ under the irradiation with the AGE light only are shown in Figure 3. Both spectra consist of near band-edge (NBE) luminescence $(3.47 \mathrm{eV})$, shallow donor (oxygen) to valence band transition $\left(I_{o x}\right.$ at $\left.3.41 \mathrm{eV}\right)$, donor acceptor pair (DAP) transition $(3.27 \mathrm{eV})$, and yellow luminescence $(\mathrm{YL})(2.3 \mathrm{eV})$ peaks. Same type of emission spectra has

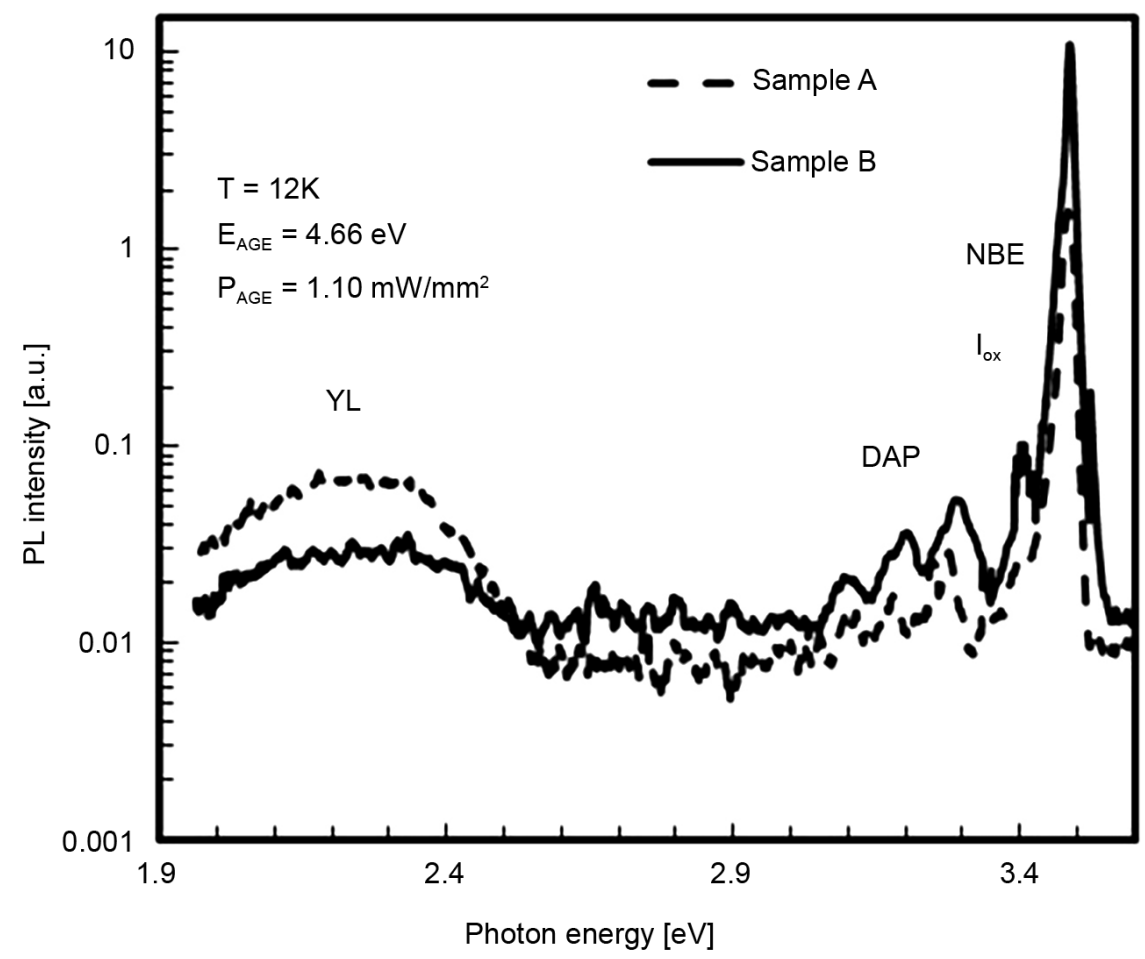

Figure 3. PL spectra of $n-G a N$ samples grown on LT-GaN buffer layer (Sample A) and AlN buffer layer (Sample B). 
been also reported for GaN in earlier studies [2]. The NBE luminescence peak intensity of sample B is 8 times higher than that of sample A while the YL intensity of sample B is lower. The PL intensity is used to measure the quality of samples, and here it elucidates that quality of sample B is better than that of the sample A.

\subsection{TWEPL Measurement}

In this study, we focus on the NBE luminescence as the principal component for comparative analysis of two samples. The normalized PL intensity $\left(I_{N}\right)$ of the NBE peak has been measured at a fixed temperature of $12 \mathrm{~K}$ and the AGE density of $1.10 \mathrm{~mW} / \mathrm{mm}^{2}$ is shown in Figure 4 as a function of the BGE power density for both samples.

With the addition of the BGE light, the value of $I_{N}$ quenches from unity for all BGE energies of $0.93,1.17$ and $1.27 \mathrm{eV}$. The quenching of the $I_{N}$ can be explained by two levels model schematically shown in Figure 5 indicating the presence of a pair of NRR centers in the sample whose energy difference correspond to that of the BGE energies [22] [23] [24] [25]. Determination of the NRR parameters becomes possible quantitatively by fitting experimental results based on Shockley-Read-Hall (SRH) statistics [22] [26].

From Figure 4, it is observed that the amount of PL quenching becomes pronounced with increasing the BGE energy and density. It also exhibits that the value of $I_{N}$ for sample A is always lower than that of sample B throughout the

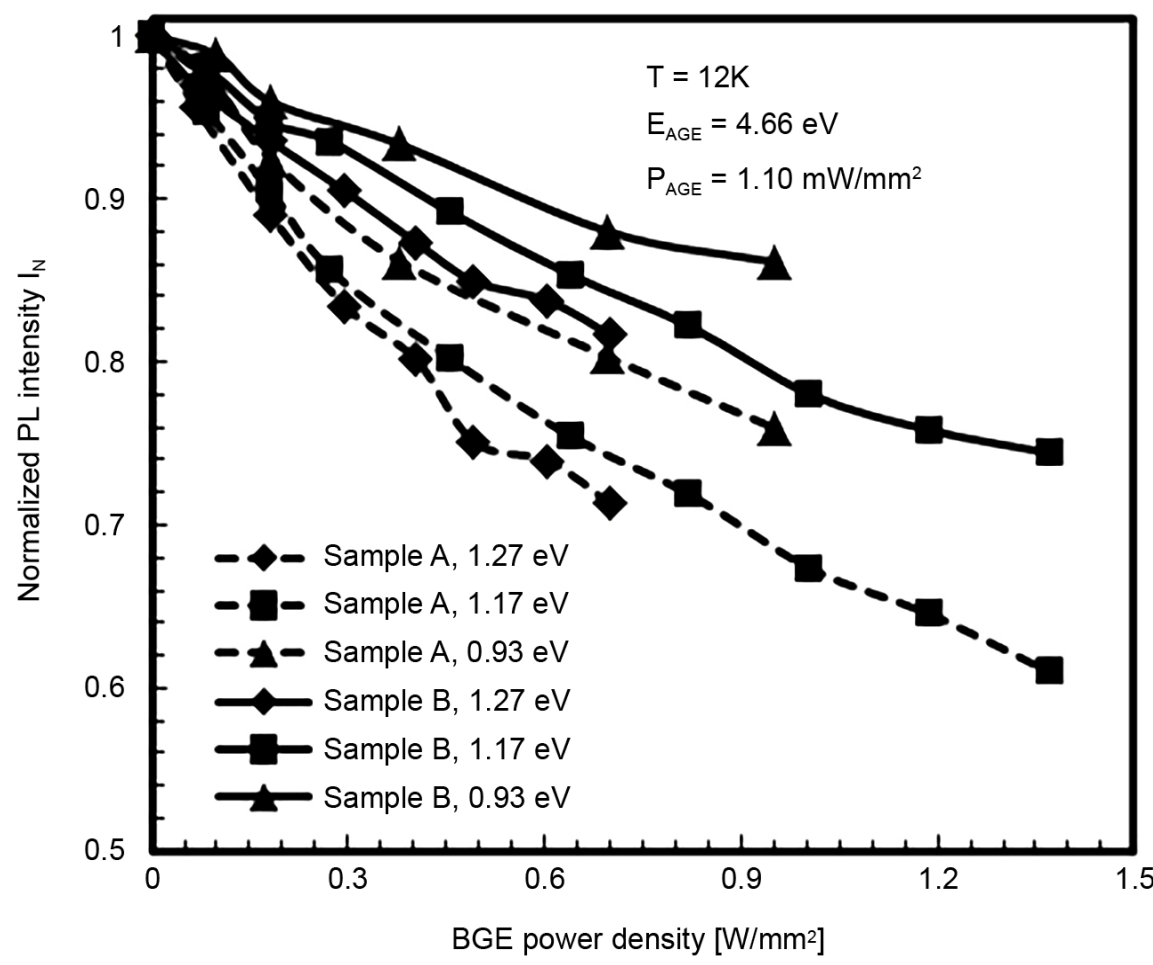

Figure 4. The normalized PL intensity $\left(I_{N}\right)$ of the NBE emission as a function of the BGE power density. 


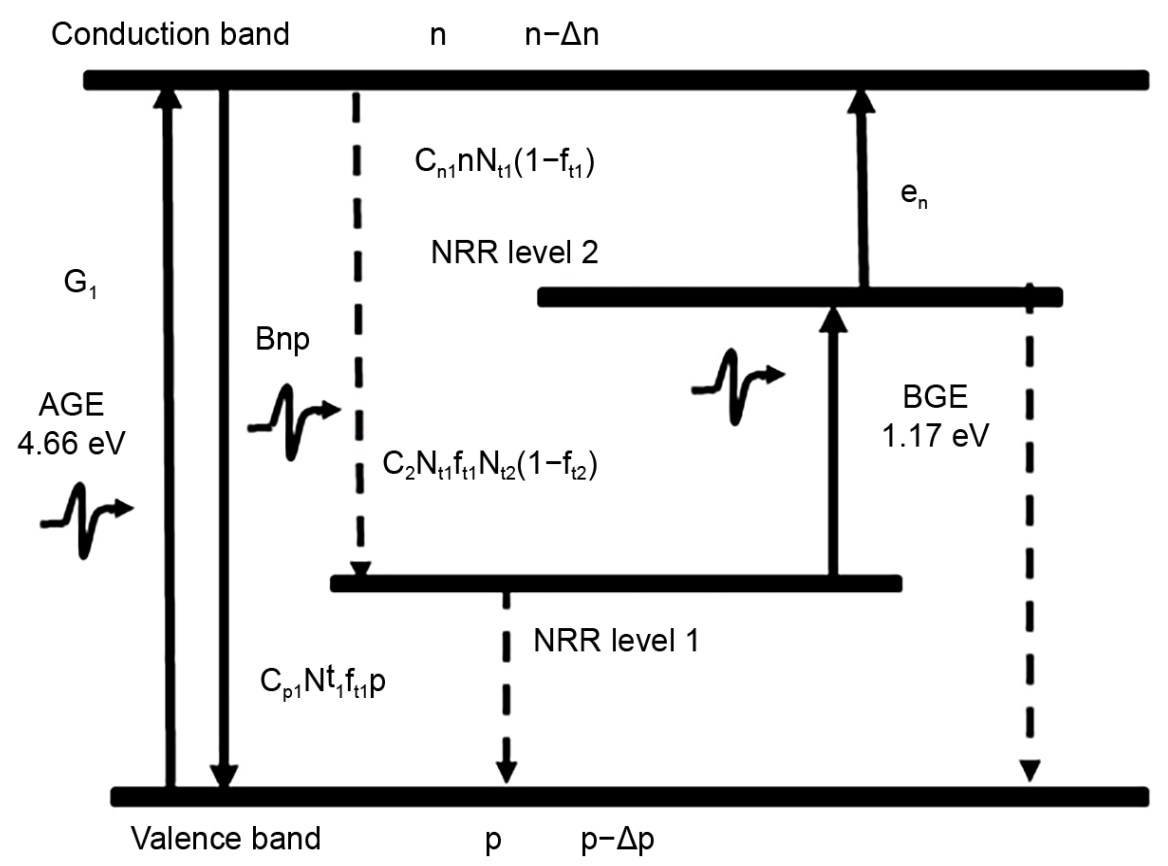

Figure 5. Two levels model of NRR process which explains the PL intensity quenching after irradiation of the BGE.

experimental BGE density range. The minimum values of $I_{N}, 0.60$ and 0.74 are obtained for samples A and B, respectively at highest BGE density $\left(1.37 \mathrm{~W} / \mathrm{mm}^{2}\right)$ of $1.17 \mathrm{eV}$ BGE. The degree of change of the $I_{N}$ values from unity represents the density of NRR centers in the samples. Thus, this result implies that the density of NRR centers is higher in sample A compared to that in sample B. It is consistent with the relative intensity of conventional PL for these samples.

When the BGE energy matches the energy difference between two coexisting below-gap NRR levels, electrons in NRR level 1 are excited to NRR level 2 from which they recombine nonradiatively with holes in the valence band of GaN. Hence, the hole density in the valence band decreases. Similarly, the electron vacancies in the NRR level 1 allow an increase of NRR process from conduction band. Thus, the electron density in the conduction band decreases. The combination of both effects reduces the number of electron-hole pairs available for radiative recombination and resulting in the PL intensity quenching. In the region of low BGE densities, the electron occupation function of NRR level 2 remains much lower than 1 and the PL quenching proceeds with the increase in the BGE density. In the region of higher BGE densities, on the other hand, the electron occupation function of NRR level 2 approaches unity and the PL quenching shows saturation tendency with further increase in the BGE density.

The AGE density dependence of $I_{N}$ has been measured at a fixed BGE density and temperature by utilizing BGE energies of 0.93 and $1.17 \mathrm{eV}$ shown in Figure 6. With increasing the AGE density from $1.10 \mathrm{~mW} / \mathrm{mm}^{2}$ to $4.60 \mathrm{~mW} / \mathrm{mm}^{2}$, the value of $I_{N}$ approaches to unity for both samples. At lower AGE density, the excitation of electrons via below gap states relative to band-to-band excitation 


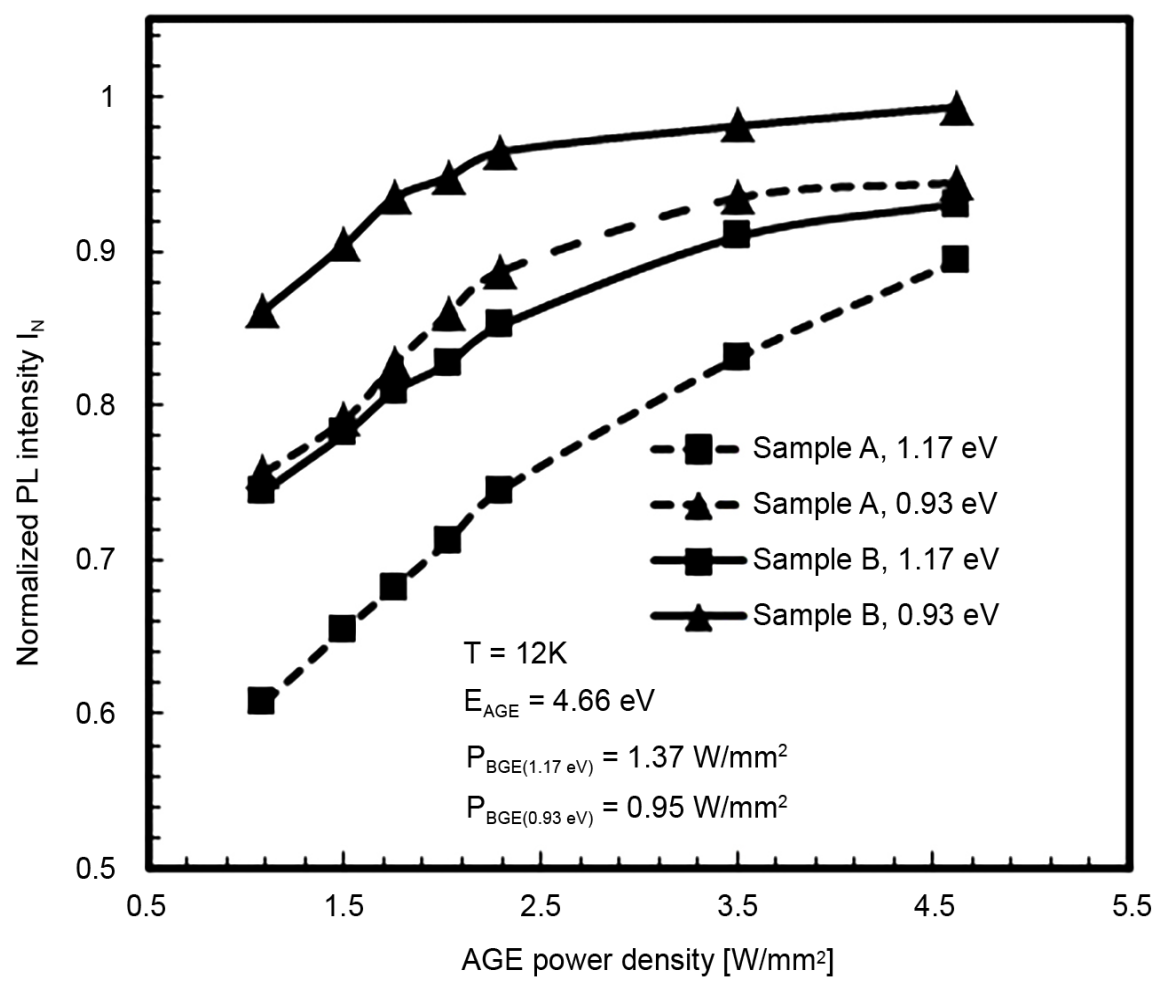

Figure 6. AGE power density dependence of the normalized PL intensity $\left(I_{N}\right)$ for samples $\mathrm{A}$ and $\mathrm{B}$.

increases which results in higher BGE effect due to the enhancement of the non-radiative recombination. Similar AGE density dependence of the $I_{N}$ was observed in our previous studies of TWEPL [22] [25] [26] [27].

The temperature dependence of $I_{N}$ for samples A and B has been also examined at a fixed AGE $\left(1.10 \mathrm{~mW} / \mathrm{mm}^{2}\right)$ and $\mathrm{BGE}\left(1.37 \mathrm{~W} / \mathrm{mm}^{2}\right.$ and 0.95 $\mathrm{W} / \mathrm{mm}^{2}$ ) densities shown in Figure 7. It has been observed that the $I_{N}$ value increases for both 1.17 and $0.93 \mathrm{eV} \mathrm{BGE}$, with increasing temperature from $12 \mathrm{~K}$ to $70 \mathrm{~K}$. The $I_{N}$ value of sample A enhances from 0.60 to 0.90 , and that of sample B from 0.74 to 0.91 , for $1.17 \mathrm{eV}$ BGE. Further increase in temperature brings little change in the $I_{N}$ values, showing a saturating tendency up to $130 \mathrm{~K}$. This type of temperature dependency was observed in previous studies and attributed to the thermal emission of electrons $e_{n}$ from NRR level 2 to the conduction band in Figure 5 in the two levels model [28] [29]. This type of thermal emission reduces the electronic population in the NRR level 2 even under the below-gap excitation and decreases the BGE effect.

\subsection{Rate Equation Analysis}

In order to corroborate our qualitative interpretation by the two levels model, a semi-quantitative simulation for the TWEPL results of $1.17 \mathrm{eV}$ BGE energy has been carried out. The rate equations of the two levels model as shown in Figure 5 can be written below with charge neutrality condition (CNC) [22] [30] [31]. 


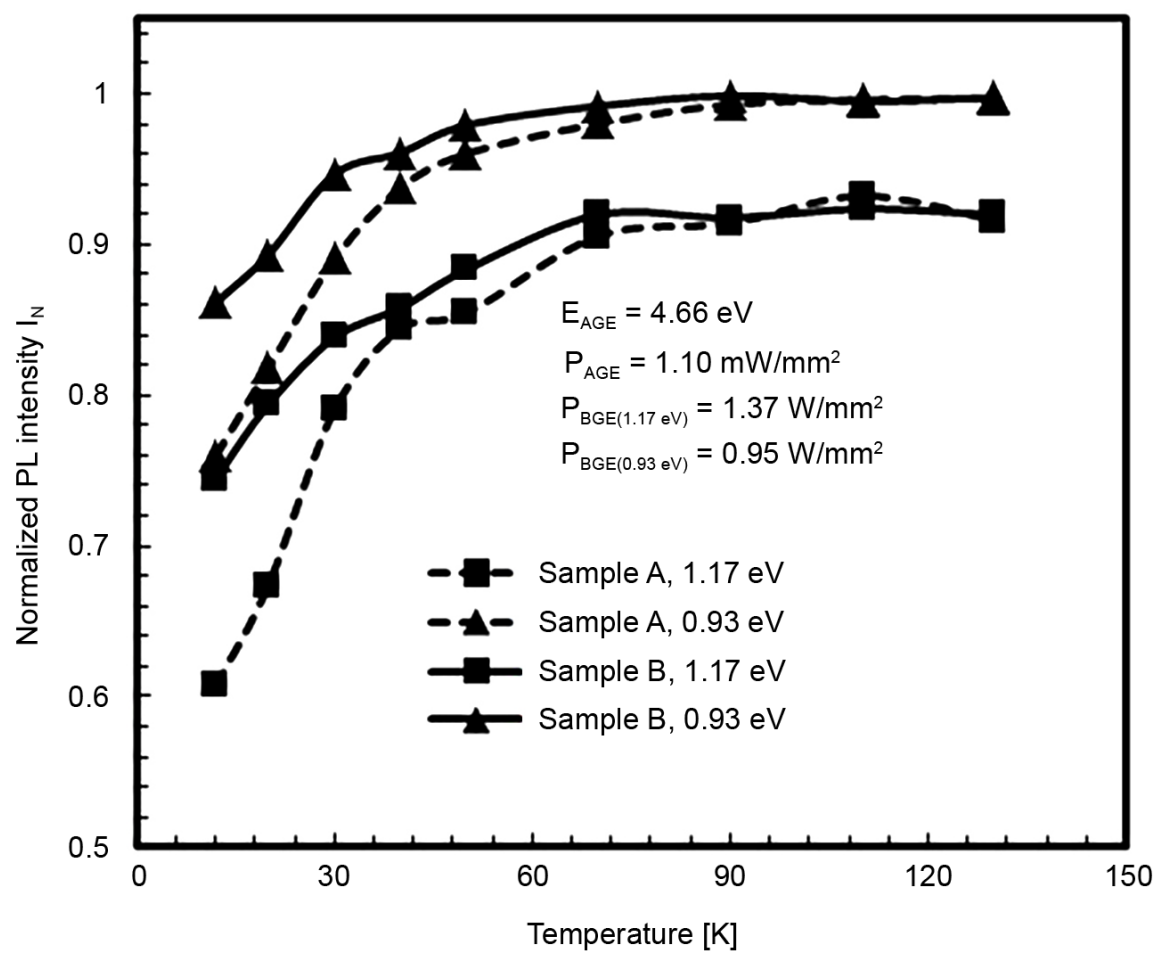

Figure 7. The Normalized PL intensity $\left(I_{N}\right)$ as a function of temperature observed for samples A and B.

$$
\begin{aligned}
& \frac{\mathrm{d} n}{\mathrm{~d} t}=G_{1}-B n p-C_{n 1} n N_{t 1}\left(1-f_{t 1}\right)=0 \\
& \frac{\mathrm{d} p}{\mathrm{~d} t}=G_{1}-B n p-C_{p 1} p N_{t 1} f_{t 1}-C_{p 2} p N_{t 2} f_{t 2}=0 \\
& \frac{\mathrm{d} f_{t 1}}{\mathrm{~d} t}=C_{n 1} n N_{t 1}\left(1-f_{t 1}\right)-C_{p 1} p f_{t 1}-G_{2} f t_{1} N_{t 2}\left(1-f_{t 2}\right)=0 \\
& \frac{\mathrm{d} f_{t 2}}{\mathrm{~d} t}=G_{2} f_{t 1} N_{t 2}\left(1-f_{t 2}\right)-C_{p 2} p N_{t 2} f_{t 2}=0 \\
& n+N_{t 1} f_{t 1}+N_{t 2} f_{t 2}=n_{0}+p
\end{aligned}
$$

where $G_{1}\left[\mathrm{~cm}^{-3} \cdot \mathrm{s}^{-1}\right]$ and $G_{2}\left[\mathrm{~cm}^{3} \cdot \mathrm{s}^{-1}\right]$ are generation rate for the AGE and the BGE, respectively, $B\left[\mathrm{~cm}^{3} \cdot \mathrm{s}^{-1}\right]$ is the radiative recombination coefficient, $N_{t}$ is the density of NRR levels, $n_{0}$ is the density of free electrons, $f_{t 1}$ and $f_{t 1}$ are the electron occupation function of NRR level 1 and NRR level 2, respectively.

For simplicity, we assumed that the electron capture coefficient $C_{n 1}$ is equal to radiative recombination coefficient $B$, as $1.2 \times 10^{-11} \mathrm{~cm}^{3} \cdot \mathrm{s}^{-1}$ for $\mathrm{GaN}$ [32]. Such consideration has been taken by other researchers [26] [33]. Reshchikov et al. [1] [34] have reported that the hole capture coefficient of $C_{p 1}$ is in the order of $10^{-6}$ $\mathrm{cm}^{3} \cdot \mathrm{s}^{-1}$ for $\mathrm{GaN}$. The hole capture coefficient of $C_{p 2}$ has been also reported in the order of $10^{-9} \mathrm{~cm}^{3} \cdot \mathrm{s}^{-1}$ [35]. The procedure of estimating generation rate of the AGE $\left(G_{1}\right)$ and BGE $\left(G_{2}\right)$ have been explained in our earlier study [30].

The density of free electrons is assumed as $n_{0} \approx 1 \times 10^{16} \mathrm{~cm}^{-3}$ for both samples considering the Si doping concentration. The system of rate equations can be solved numerically and the dependencies of $n, p, f_{t 1}$, and $f_{t 2}$ on $G_{2}$ was found for 
the constant parameters of $G_{1}, B, n_{0}, N_{t} C_{n}$, and $C_{p}$. By systematically solving and fitting the simulated results with experimental data, the defect parameters have been chosen as $G_{1}=4.0 \times 10^{20} \mathrm{~cm}^{-3} \cdot \mathrm{s}^{-1}, C_{p 1}=1 \times 10^{-6} \mathrm{~cm}^{3} \cdot \mathrm{s}^{-1}, C_{n 1}=8.5 \times$ $10^{-11} \mathrm{~cm}^{3} \cdot \mathrm{s}^{-1}$ and $C_{p 2}=6.5 \times 10^{-9} \mathrm{~cm}^{3} \cdot \mathrm{s}^{-1}$ for both samples A and B, respectively. The densities of two NRR levels are obtained as $N_{t 1}=8.0 \times 10^{15} \mathrm{~cm}^{-3}, N_{t 2}=3.0 \times$ $10^{17} \mathrm{~cm}^{-3}$ for the sample A, and $N_{t 1^{\prime}}=6.1 \times 10^{15} \mathrm{~cm}^{-3}, N_{t 2^{\prime}}=6.0 \times 10^{16} \mathrm{~cm}^{-3}$ for the sample B. The value of $I_{N}$ is calculated as a function of generation rate of BGE $\left(G_{2}\right)$ under fixed AGE generation rate of $4.0 \times 10^{20} \mathrm{~cm}^{-3} \cdot \mathrm{s}^{-1}$ and shown in Figure 8. The broken and solid lines represent the simulated result together with experimental points for both samples. The simulated $I_{N}$ value shows a reasonable agreement with the measured points.

The dependence of the $I_{N}$ as a function of the electron-hole generation rate of the AGE $\left(G_{1}\right)$ at $12 \mathrm{~K}$ has been calculated by setting $G_{2}=1.0 \times 10^{-14} \mathrm{~cm}^{3} \cdot \mathrm{s}^{-1}$ and keeping all the other parameters as constant as previous. Figure 9 shows the $I_{N}$ value of the NBE peak for both samples as a function of the electron-hole generation rate of the $\operatorname{AGE}\left(G_{1}\right)$.

Here, a set of parameters give the insight of below-gap states acting as NRR centers in samples $\mathrm{A}$ and $\mathrm{B}$, and a reasonable fitting with experimental data. The estimated result shows that the densities of NRR centers are lower in sample B than that in sample A. From both fitting results, it is concluded that the interpretation based on the two-levels model is valid and the use of the AlN buffer layer is more effective for reducing the density of NRR centers in $\mathrm{n}-\mathrm{GaN}$ layer than the LT-GaN buffer layer. The TWEPL study of NRR centers guides us to optimize growth conditions further.

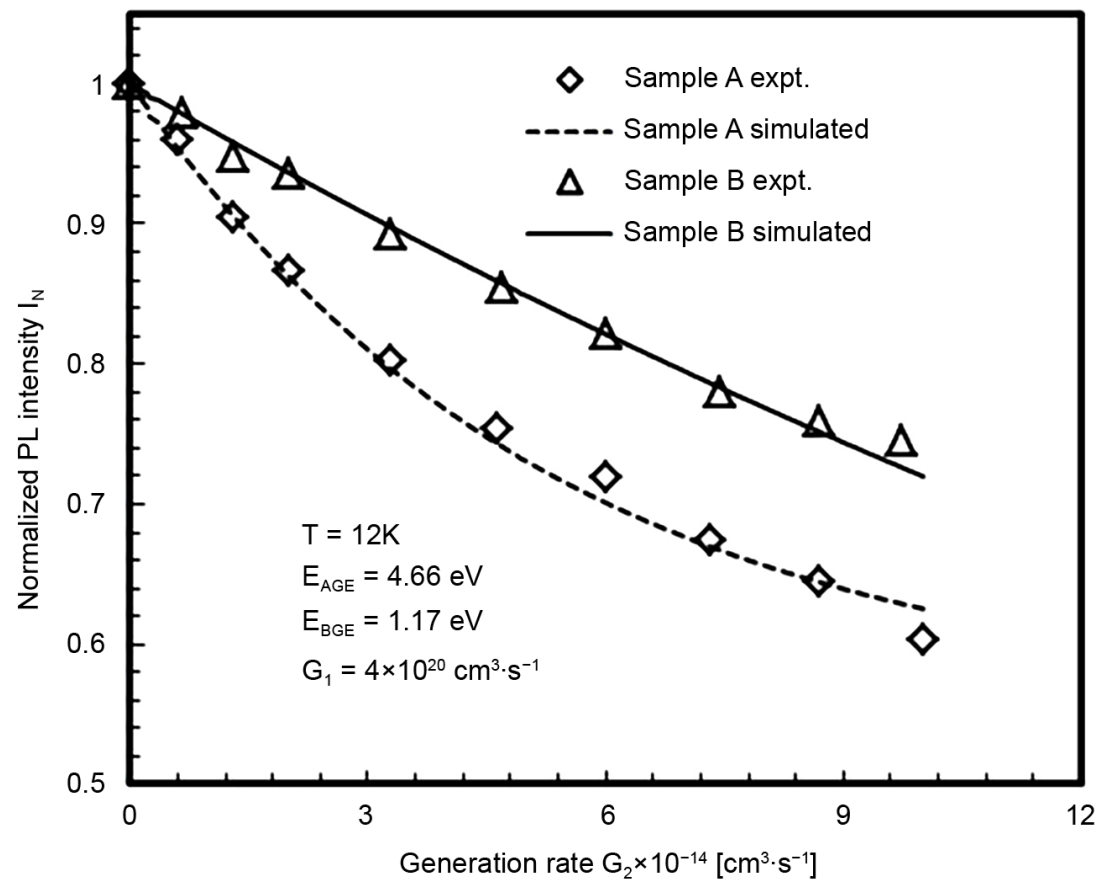

Figure 8. Variation of the normalized PL intensity as a function of BGE density $\left(G_{2}\right)$. The broken and solid lines represent the simulated results for sample A and B, respectively. 


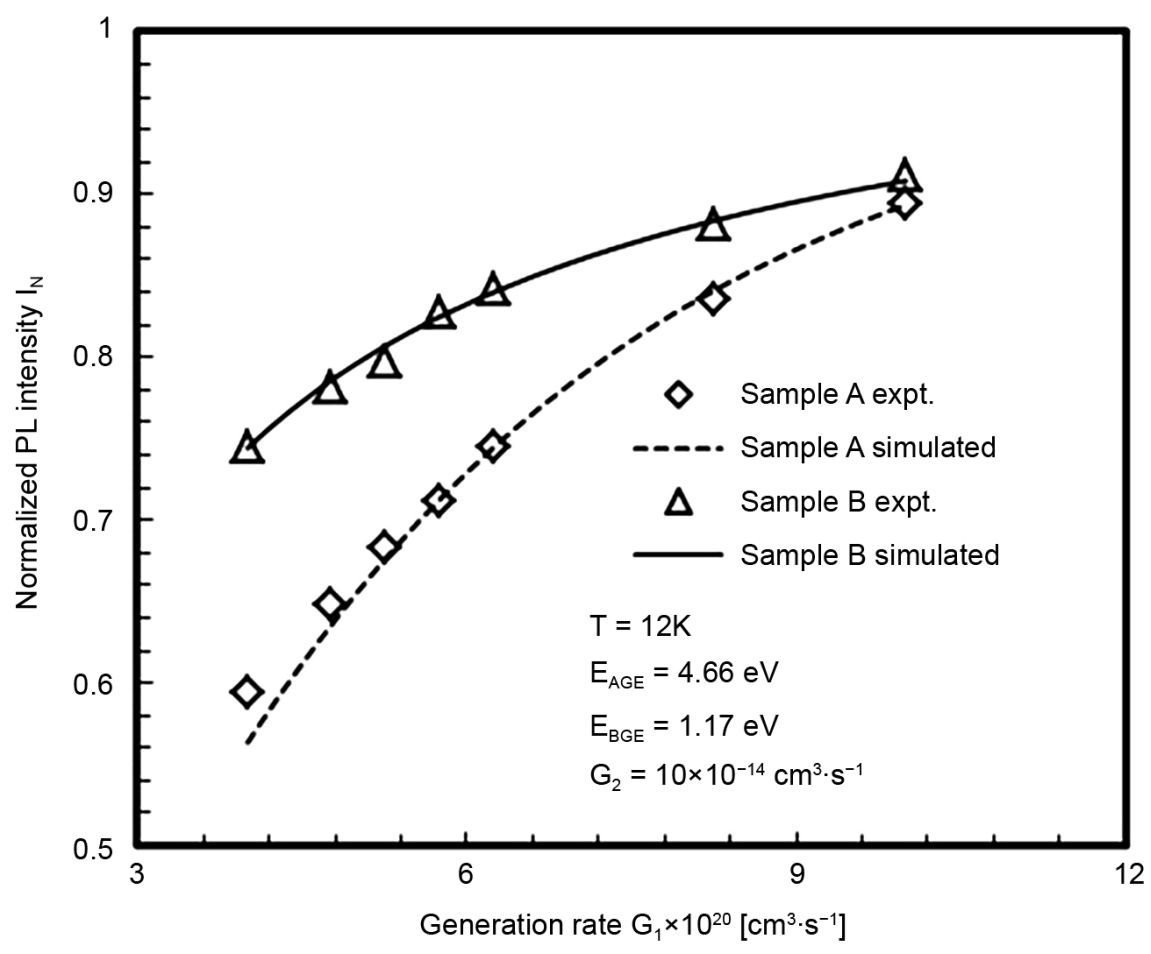

Figure 9. AGE density $\left(G_{1}\right)$ dependence of the normalized PL intensity in samples A and B. The broken and solid lines represent the simulated results.

\section{Conclusion}

Defect States acting as NRR centers in n-type GaN layers grown on a LT-GaN buffer layer and aAlN buffer layer have been studied by TWEPL method. The near band-edge PL peak intensity quenches after the irradiation of BGE energies of $0.93,1.17$ and $1.27 \mathrm{eV}$. The quenching of the PL intensity has been interpreted by the two levels model and indicates the presence of a pair of NRR centers in the samples which are activated by the BGE. The dominant quenching of the PL intensity for the sample A (with LT-GaN buffer layer) indicates a direct evidence for the higher density of NRR centers compared to the sample B (with AlN buffer layer). A simulation of rate equations agreed well with our experimental data with a set of NRR parameters. The use of AlN buffer layer is more effective for reducing the NRR density in $\mathrm{n}-\mathrm{GaN}$ layers than the LT-GaN buffer layer.

\section{References}

[1] Monemar, B. (1999) III-V Nitrides-Important Future Electronic Materials. Journal of Materials Science: Materials in Electronics, 10, 227-254. https://doi.org/10.1023/A:1008991414520

[2] Reshchikov, M.A. and Morkoc, H. (2005) Luminescence Properties of Defects in GaN. Journal of Applied Physics, 97, 061301. https://doi.org/10.1063/1.1868059

[3] Yu, H., Caliskan, D. and Ozybay, E. (2006) Growth of High Crystalline Quality Semi-Insulating GaN Layers for High Electron Mobility Transistor Applications. Journal of Applied Physics, 100, 033501. https://doi.org/10.1063/1.2221520

[4] Lester, S.D., Ponce, F.A., Craford, M.G. and Steigerwald, D.A. (1995) High Disloca- 
tion Densities in High Efficiency GaN-Based Light-Emitting Diodes. Applied Physics Letter, 66, 1249-1251. https://doi.org/10.1063/1.113252

[5] Weimann, N.G., Eastman, L.F., Doppalapudi, D., Ng, H.M. and Moustakas, T.D. (1998) Scattering of Electrons at Threading Dislocations in GaN. Journal of Applied Physics, 83, 3656-3659. https://doi.org/10.1063/1.366585

[6] Cao, X.A., Teetsov, J.A., Sandvik, F.S. and Arthur, S.D. (2004) Microstructural Origin of Leakage Current in GaN/InGaN Light-Emitting Diodes. Journal of Crystal Growth, 264, 172-177. https://doi.org/10.1016/j.jcrysgro.2004.01.031

[7] Guha, S., Depuydt, J.M., Haase, M.A., Qiu, J. and Cheng, H. (1993) Degradation of II-VI Base Blue-Green Light Emitters. Applied Physics Letter, 63, 3107-3109. https://doi.org/10.1063/1.110218

[8] Sugahara, T., Sato, H., Hao, M., Naoi, Y., Kurai, S., Tottori, S., Yamashita, K., Nishino, K., Romano, L.T. and Sakai, S. (1998) Direct Evidence That Dislocations Are Nonradiative Recombination Centers in GaN. Japanese Journal of Applied Physics, 37, L398-L400.

[9] Rosner, S.J., Carr, E.C., Ludowise, M.J., Girolami, G. and Erikson, H.I. (1997) Correlation of Cathodoluminescence Inhomogeneity with Microstructural Defects in Epitaxial GaN Grown by Metal Organic Chemical Vapor Deposition. Applied Physics Letter, 70, 420-422. https://doi.org/10.1063/1.118322

[10] Yoshida, S., Misawa, S. and Gonda, S. (1983) Improvements on the Electrical and Luminescent Properties of Reactive Molecular Beam Epitaxially Grown GaN Films by Using AlN Coated Sapphire Substrates. Applied Physics Letter, 42, 427-429. https://doi.org/10.1063/1.93952

[11] Kuznia, J.N., Khan, M.A., Olson, D.T., Kaplan, R. and Freitas, J. (1993) Influence of Buffer Layers on the Deposition of High Quality Single Crystal GaN over Sapphire Substrates. Journal of Applied Physics, 73, 4700-4702. https://doi.org/10.1063/1.354069

[12] Coreki, S., Ozturk, M.K., Bengi, A., Cakmak, M., Ozcelik, S. and Ozbay, E. (2011) Charaterization of an AlN Buffer Layer and a Thick-GaN Layer Grown on Sapphire Substrate by Metal Organic Chemical Vapor Deposition (MOCVD). Journal of Material Science, 46, 1606-1612. https://doi.org/10.1007/s10853-010-4973-7

[13] Amano, H., Sawaki, N., Akasaki, I. and Toyoda, Y. (1986) Metalorganic Vapor Phase Epitaxial Growth of High Quality GaN Film Using an AlN Buffer Layer. Applied Physics Letter, 48, 353-355. https://doi.org/10.1063/1.96549

[14] Jeon, H.C., Lee, S.J., Kumar, S., Kang, T.W., Lee, N.H. and Kim, T.W. (2014) Effect of AlN Buffer Layers on the Structural and Optical Properties of GaNEpilayer Grown on $\mathrm{Al}_{2} \mathrm{O}_{3}$ Substrates by Using Plasma Assisted Molecular Beam Epitaxy. Journal of Korean Physical Society, 64, 1128-1131.

https://doi.org/10.3938/jkps.64.1128

[15] Amano, H., Iwaya, M., Kashima, T., Katsuragawa, M., Akasaki, I., Han, J., Hearne, S., Floro, J.A., Chason, E. and Figile, J. (1998) Stress and Defect Control in GaN Using Low Temperature Interlayers. Japanese Journal of Applied Physics, 37, L1540-L1542. https://doi.org/10.1143/JJAP.37.L1540

[16] Muhtadi, S., Hwang, S., Coleman, A., Asif, F., Lunev, A., Chandrashekhar, M.V.S. and Khan, A. (2017) High Temperature Operation of $n$-AlGaN Channel Metal Semiconductor Field Effect Transistors on Low-Defect AlN Templates. Applied Physics Letters, 110, Article ID: 193501. https://doi.org/10.1063/1.4982656

[17] Fleischmann, S., Richter, E., Mogilatenko, A., Weyers, M. and Tränkle, G. (2017) Influence of AlN Buffer Layer on Growth of AlGaN by HVPE. Physica Status Solidi 
(B), 254, Article ID: 1600696. https://doi.org/10.1002/pssb.201600696

[18] Detchprohm, T., Yano, M., Sano, S., Nakamura, R., Mochiduki, S., Nakamura, T., Amano, H. and Akasaki, I. (2001) Heteroepitaxial Lateral Overgrowth of GaN on Periodically Grooved Substrates a New Approach for Growing Low-Dislocation-Density GaN Single Crystals. Japanese Journal of Applied Physics, 40, L16-L19. https://doi.org/10.1143/JJAP.40.L16

[19] Yang, J.H., Kang, S.M., Dinh, D.V. and Yoon, D.H. (2009) Influence of AlN Buffer Layer Thickness and Deposition Methods on GaN Epitaxial Growth. Thin Solid Films, 517, 5057-506. https://doi.org/10.1016/j.tsf.2009.03.089

[20] Wang, C.D., Yu, L.S., Lau, S.S., Yu, E.T., Kim, W., Botchkarev, A.E. and Morkoc, H. (1998) Deep Level Defects in $n$-Type GaN Grown by Molecular Beam Epitaxy. Applied Physics Letters, 72, 1211-1213. https://doi.org/10.1063/1.121016

[21] Gotz, W., Johnson, N.M., Amano, H. and Akasaki, I. (1994) Deep Level Defects in n-Type GaN. Applied Physics Letters, 65, 463-465.

[22] Kamata, N., Ocampo, J.M.Z., Hoshino, K., Yamada, K., Nishioka, M., Someya, T. and Arakawa, Y. (1999) Below-Gap Spectroscopy of Semiconductor Quantum Wells by Two Wavelength Excited Photoluminescence. Recent Research Development on Quantum Electronics, 1, 123-135.

[23] Grimmeiss, H.G. and Monemar, B. (1973) Some Optical Properties of Cu in GaP. Physica Status Solidi $(A)$, 19, 505-511. https://doi.org/10.1002/pssa.2210190214

[24] Tajima, M. (1984) Fatigue and Recovery Effects of the 0.65-eV Emission Band in GaAs. Japanese Journal of Applied Physics, 23, L690-L693. https://doi.org/10.1143/JJAP.23.L690

[25] Touhidul Islam, A.Z.M., Murakoshi, N., Fukuda, T., Hirayama, H. and Kamata, N. (2014) Optical Detection of Nonradiative Recombination Centers in AlGaN Quantum Wells for Deep UV Region. Physica Status Solidi (C), 11, 832-835. https://doi.org/10.1002/pssc.201300405

[26] Julkarnain, M., Fukuda, T., Kamata, N. and Arakawa, Y. (2015) A Direct Evidence of Allocating Yellow Luminescence Band in Undoped GaN by Two-Wavelength Excited Photoluminescence. Applied Physics Letters, 107, Article ID: 212102. https://doi.org/10.1063/1.4936243

[27] Ocampo, J.M.Z., Klausing, Z., Semchinova, H.O., Stemmer, J., Hirasawa, M., Kamata, N. and Yamada, K. (2001) Study of MBE-Grown GaN/AlGaN Quantum Well Structures by Two-Wavelength Excited Photoluminescence. Physica Status Solidi (A), 183, 189-195. https://doi.org/10.1002/1521-396X(200101)183:1<189::AID-PSSA189>3.0.CO;2-5

[28] Kamata, N., Klausing, H., Fedler, F., Mistele, D., Aderhold, J., Semchinova, O.K., Graul, J., Someya, T. and Arakawa, Y. (2004) Effect of Modulation-Doping on Luminescence Properties of Plasma Assisted MBE-Grown GaN/AlGaN Quantum Well. European Physical Journal of Applied Physics, 27, 271-273.

[29] Klausing, H., Kamata, N., Takahashi, F., Fedler, F., Mistele, D., Aderhold, J., Semchinova, O.K., Graul, J., Someya, T. and Arakawa, Y. (2003) Improved Quality of Plasma Assisted MBE-Grown GaN/AlGaN Quantum Wells Revealed by Two-Wavelength Excited Photoluminescence. Physica Status Solidi (C), 2658-2661. https://doi.org/10.1002/pssc.200303473

[30] Shockley, W. and Read, W.T. (1952) Statistics of the Recombinations of Holes and Electrons. Physical Review, 87, 835-842. https://doi.org/10.1103/PhysRev.87.835

[31] Hall, R.N. (1952) Electron-Hole Recombination in Germanium. Physical Review, 87, 387. https://doi.org/10.1103/PhysRev.87.387 
[32] Piprek, J. (2010) Efficiency Droop in Nitride-Based Light-Emitting Diodes. Physica Status Solidi $(A), 207,2217-2225$. https://doi.org/10.1002/pssa.201026149

[33] Grieshaber, W., Schubert, E.F., Goepfert, I.D., Karlicek, R.F., Schurman Jr., M.J. and Tran, C. (1996) Competition between Band Gap and Yellow Luminescence in GaN and Its Relevance for Optoelectronic Devices. Journal of Applied Physics, 80, 4615-4620. https://doi.org/10.1063/1.363443

[34] Reschikov, M.A. and Korotkov, R.Y. (2001) Analysis of the Temperature and Excitation Intensity Dependencies of Photoluminescence in Undoped GaN Films. Physical Review B, 64, Article ID: 115205.

[35] Kamata, N., Ocampo, J.M.Z., Okamoto, W., Hoshino, K., Someya, T., Arakawa, Y. and Yamada, K. (2002) Below-Gap Recombination Dynamics in GaN Revealed by Time-Resolved and Two-Wavelength Excited Photoluminescence. Materials Science and Engineering. B, 91-92, 290-293. https://doi.org/10.1016/S0921-5107(01)01032-7 